\title{
Spinocerebellar Ataxia Type 23: A Genetic Update
}

\author{
Dineke S. Verbeek
}

Published online: 17 December 2008

(C) The Author(s) 2008. This article is published with open access at Springerlink.com

\begin{abstract}
The spinocerebellar ataxia type 23 locus was identified in 2004 based on linkage analysis in a large, twogeneration Dutch family. The age of onset ranged 4356 years and the phenotype was characterized by a slowly progressive, isolated ataxia. Neuropathological examination revealed neuronal loss in the Purkinje cell layer, dentate nuclei, and inferior olives. Ubiquitin-positive intranuclear inclusions were found in nigral neurons, but were considered to be Marinesco bodies. The disease locus on chromosome 20p13-12.3 was found to span a region of approximately $6 \mathrm{Mb}$ of genomic DNA, containing 97 known or predicted genes. To date, no other families have been described that also map to this SCA locus. Direct sequencing of the coding regions of 21 prioritized candidate genes did not reveal any disease-causing mutation. Apparently, the SCA23 gene is a disease gene with a different function than the genes that have been associated with other known SCA types. Work to elucidate the chromosomal organization of the SCA23 locus will eventually discover the responsible disease gene.
\end{abstract}

\section{Introduction}

Autosomal dominant cerebellar ataxias (ADCA) comprises a clinically and genetically heterogeneous group of neurodegenerative disorders characterized by a cerebellar ataxic syndrome. As most of the disease phenotypes no longer fit

D. S. Verbeek $(\triangle)$

Department of Genetics, University Medical Center Groningen,

University of Groningen,

P.O. Box 30 001, 9700 RB Groningen, The Netherlands

e-mail: D.S.Verbeek@medgen.umcg.nl into the categories defined by Harding [1], ADCA is now classified by the causal mutation or gene locus. So far 26 different subtypes of ADCA have been recognized and are known as the spinocerebellar ataxias (SCA1-8, 10-29) [2, 3]. The SCA9 locus has been reserved since 2001 but there is still no publication about it, while SCA19 and SCA22 may be allelic diseases. However, the disease gene and causal mutation has been identified in just 17 SCA types (SCA1-7, 10-17, 27 and 28) [2, 4-7].

The most recent SCA mutations identified are not like the first mutations, which coded CAG (glutamine) or noncoding repeat expansions (SCA1-3, 6-8, 10, 12, and 17) [2]; rather they are missense, deletion or duplication mutations (SCA14, 4-5, 11, 13-16, 20 and 28) [2, 5-8]. The different disease-causing mechanisms have changed the view on the pathology of the disorder, as it is no longer considered to be a "pure" polyglutamine disorder, such as Huntington's disease or Kennedy's disease. The fact that different mutational mechanisms in various disease genes lead to similar disease phenotypes strongly suggests that common biological pathways must underlie the different SCA types.

Worldwide, SCA3 mutations display a prevalence of 20 $25 \%$, but there are regional variations $[9,10]$. SCA1, 2, 6, and 7 , on average displayed a prevalence of approximately $2-5 \%$ [11]. However, the SCA1 mutation is most frequently identified in Africa [12], while SCA2 accounts for most of the cases in India, Cuba, and Italy [13, 14]. The remaining SCA types are considered to be rare mutations, identified in singular families or specific populations, and do not explain the majority of the unclassified SCA cases. Thus, the heterogeneity of the disorder is reflected by how the prevalence varies between different ethnic and continental groups and moreover indicates that novel SCA genes still need to be found. 
In 2004, Verbeek et al. reported a two-generation Dutch ADCA family with a slowly progressive, isolated ataxia [15], with no mutations in the SCA genes known then (SCA1-3, 6-8, 12 and 17). Genome-wide linkage analysis in 14 family members mapped the new disease gene to chromosome region 20p13-12.3. As this region was not known to be involved in ADCA, their disorder was designated as spinocerebellar ataxia type 23 (SCA23) by the Human Genome Nomenclature Committee.

\section{Clinical Observations}

The pattern of disease transmission in the SCA23 family was consistent with an autosomal dominant inheritance. There were no affected individuals of the first generation still alive at the time of the assessment, so no clinical data could be obtained. The presence of clinical anticipation could not therefore be confirmed. In the 2004 study, we examined five second-generation individuals with a mean age of onset of 50.4 years (range 43-56 years) and a mean duration of clinical symptoms at examination of 10.2 years (range 1-23 years). Based on these data, the disease progression seemed to be relatively slow and the disease severity correlated with the duration of the disease, as wheelchair dependence only occurred 23 years after onset of the disease.

The initial symptom in three patients was gait ataxia and in two of the patients it was cycling difficulties and a concurrent decline of gait and speech. In these latter patients, gait ataxia followed somewhat later after the first symptoms presented. All patients showed a relatively slowly progressive, isolated, cerebellar ataxia without any distinctive features such as cognitive deterioration, epilepsy, extrapyramidal features, or peripheral nerve involvement. Additional neurological examination revealed dysarthria, oculomotor problems such as slowing saccades and ocular dysmetria, and decreased vibration sense below the knee in three affected individuals. Four patients showed hyperreflexia and two displayed Babinski's signs.

Not much is known on the clinical variability as only one family has been described. SCA23 may only have a few symptoms besides the gait ataxia and is referred to as a "pure" ataxia, similar to SCA5-6, 11, 15-16, and 22 [11].

\section{Neuropathology}

Macroscopic examination of the brain of one SCA23 patient showed marked atrophy of the frontotemporal region and vermis of the cerebellum, the basis pontis, and the spinal cord. The weight of the brain $(930 \mathrm{~g})$ reflected the large loss of tissue due to the disease. Pronounced neuronal loss was identified in the Purkinje cell layer of the rostal vermis, the dentate nuclei and inferior olives; the surrounding white matter showed myelin loss and gliosis. Although normal myelination was seen of the white matter tracts in the basis pontis, the cerebellopontine tracts were relatively small. No clear loss of motor neurons was observed in the spinal cord.

Neuropathological studies of the SCA types that have recently been genetically defined are rare and are often performed on the autopsies of patients who had end-stage disease. However, some reports describe detailed morphological findings on SCA1-3, 6, and 7 [11]. If we compare the pathological features of the SCA23 brain with other SCA types, it mostly closely resembles SCA6 pathology, as only marked atrophy was observed in the cerebellum with specific loss of the Purkinje cells and neuronal loss in the inferior olives. No massive cell loss was detected in the substantia nigra or pons, which is often seen in SCA1-3 pathology and is probably associated with the severity and progression of these subtypes.

Ubiquitin-positive neural inclusions ( $2-5 \mu \mathrm{m}$ in diameter) were detected in some of the nigral neurons. However, these inclusions did not resemble those protein aggregates frequently correlated with polyglutamine-induced SCA types and were considered to be Marinesco bodies. Neurons in the substantia nigra occasionally contained Lewy bodies. Additional neurodegenerative deposits observed using different immuno-histochemical stainings (e.g. Bodian silver and $\beta$ amyloid) were generally rare or absent. However, some micro- and astroglia cells in and around the central nuclei and substantia nigra were positive for tau-staining.

\section{Gene Mapping and Molecular Studies}

As regular diagnostic screening and linkage analysis excluded the known SCA genes and loci, we performed a genome-wide screen in an attempt to map the disease gene. Two-point linkage analysis revealed significant linkage with marker D20S199 (max. lod score 3.46). The candidate interval was located directly on the tip of the short arm of chromosome 20 and was flanked proximally by marker D20S1155. The region spans $18.2 \mathrm{cM}$ in size, which corresponds to approximately $6 \mathrm{Mb}$ of genomic DNA, and contains 97 predicted and known transcripts.

Genes containing a coding CTG or CAG repeat were considered to be primary candidate genes for SCA23 disease. This resulted in the identification of three genes in the SCA23 interval: GFR receptor $\alpha-4$ (GFRA4), attractin $(A T R N)$, and ubiquitin-conjugating enzyme 7 interacting protein 3 ( $R B C K 1)$. However, no polyleucine or polyglutamine repeat expansions were observed in these genes using Southern blotting. Three additional genes were 
selected based on their function, neuronal expression, and ataxic phenotype in gene-specific mutant mice, including neuronal death inducible kinase (SKIP3), major prion protein precursor $(P R N P)$, and the prion gene complex downstream $(P R N D)$. Direct sequencing of the coding regions and intron-exon boundaries of the selected genes did not reveal any disease-causing alterations.

After this exclusion, an alternative approach was taken. The notion that different mutational mechanisms in diverse genes lead to identical disease phenotypes strongly suggests that common biological pathways must underlie the different SCA types. Therefore, a protein-protein interaction network was developed for 20 different inherited cerebellar ataxias [16]. This network analysis revealed several unsuspected interactions between the diverse disease proteins and it also harbors potentially new disease candidate genes for the SCA loci in which no disease gene has been identified yet.

In order to prioritize the 97 SCA23 candidate disease genes, the protein interaction data available from the ataxia network and the Prioritizer program was used [17]. This program prioritizes candidate genes in the SCA23 interval, based on the position that these genes are given in a gene network founded on the knowledge of the known SCA genes. This resulted in the identification of three genes in the SCA23 interval that were shown to be physical interaction partners of known ataxia genes in the yeast two-hybrid screen: proteasome inhibitor PI31 subunit (PSMF1), FK506 binding protein 1A (FKBP1A), and isocitrate dehydrogenase $3(\mathrm{NAD}+)$ beta $(I D H 3 B)$ [16]. These three potential candidates were screened for mutations in the coding regions and intron-exon boundaries via direct sequencing but no mutations were found. Using the Prioritizer program, an additional 18 candidate genes from the SCA23 region were selected based on their $p$ value (the lower the $p$ value the more likely that this gene is indeed the SCA23 disease gene) and gene function (Table 1). Unfortunately, extensive sequencing of annotated exons and exon-intron boundaries did not identify any diseasecausing mutation. Twenty-four single nucleotide polymorphisms (SNPs) were detected in 17 exons of the 18 genes (data not shown). From these SNPs, only one of the variations was unknown in the Ensembl SNP database (release 50, July 2008; www.ensembl.org/snpview) and was located in exon 1 (c.136A>G) of the ADRAl gene and changed the amino acid serine to glycine. However, this change seemed to be tolerated as it was also observed in healthy controls, and additionally did not fully co-segregate with the disease phenotype within the family. This variation was not therefore considered to be a disease-causing variation.

\section{Discussion}

SCA23 has so far been identified in one Dutch ADCA family and is characterized by a slowly progressive isolated cerebellar ataxia. As no other families have been found that

Table 1 Overview of sequenced prioritized candidate genes within SCA23 interval

\begin{tabular}{|c|c|c|c|c|c|}
\hline Position prioitizer & Gene & $p$ value & $\mathrm{Chr}$ & Start & End \\
\hline 1 & SNPH & 0.0003998400639 & 20 & 1194960 & 1237969 \\
\hline 2 & GPR73L1 & 0.009196321471411436 & 20 & 5230686 & 5243015 \\
\hline 3 & NSFL1C & 0.009596161535385846 & 20 & 1370811 & 1396417 \\
\hline 4 & GFRA4 & 0.012395041983206718 & 20 & 3587939 & 3592046 \\
\hline 5 & RASSF2 & 0.013594562175129948 & 20 & 4708670 & 4752291 \\
\hline 6 & FKBP1A & 0.014394242303078768 & 20 & 1297623 & 1321745 \\
\hline 7 & ADRA1D & 0.015193922431027589 & 20 & 4149816 & 4177659 \\
\hline 8 & TGM3 & 0.015593762495002 & 20 & 2224675 & 2269725 \\
\hline 9 & AVP & 0.01599360255897641 & 20 & 3011203 & 3013370 \\
\hline 11 & SLC23A2 & 0.01639344262295082 & 20 & 4781002 & 4930145 \\
\hline 12 & PTPNS1 & 0.01719312275089964 & 20 & 1823425 & 1868532 \\
\hline 14 & CENPB & 0.021591363454618154 & 20 & 3713331 & 3715130 \\
\hline 17 & PSMF1 & 0.025189924030387844 & 20 & 1041939 & 1095971 \\
\hline 18 & PTPRA & 0.025989604158336666 & 20 & 2769373 & 2967314 \\
\hline 19 & UBOX5 & 0.025989604158336666 & 20 & 3036220 & 3088540 \\
\hline 22 & CSNK2A1 & 0.029188324670131948 & 20 & 411340 & 472482 \\
\hline 23 & ITPA & 0.03078768492602959 & 20 & 3138056 & 3152504 \\
\hline 27 & $\mathrm{CDC} 25 \mathrm{~B}$ & 0.032387045181927226 & 20 & 3724386 & 3734759 \\
\hline 30 & STK35 & 0.036785285885645745 & 20 & 2031519 & 2077196 \\
\hline 36 & SNRPB & 0.04558176729308277 & 20 & 2390281 & 2399499 \\
\hline 42 & IDH3B & 0.06717313074770093 & 20 & 2587043 & 2592853 \\
\hline
\end{tabular}


also map to this locus, additional effort must be put into following-up the individuals in the second and third generations presumed at-risk. We hope eventually to narrow down the size of the SCA23 region and limit the number of potential candidate genes. Reviewing the latest data, the question arises: where is the disease gene and its mutation? Despite the extensive molecular analysis of 24 genes that was based on good knowledge of the function and expression of the candidate genes, we have been unable to identify any disease-causing mutation. The newest genetic data even suggest that SCA23 is caused by mutations in a completely different gene than has been described for the known SCA types so far, because we did not even find mutations in the prioritized candidate genes. As two of the more recent SCA types (15 and 20) were caused by in-frame deletion and duplication mutations, it will be necessary to use additional molecular approaches, such as MLPA or array-based high-density SNP typing to elucidate the chromosomal organization at the SCA23 locus.

Acknowledgements The author wishes to thank Cisca Wijmenga and Richard Sinke for critically reading the manuscript and Jackie Senior for editing.

Open Access This article is distributed under the terms of the Creative Commons Attribution Noncommercial License which permits any noncommercial use, distribution, and reproduction in any medium, provided the original author(s) and source are credited.

\section{References}

1. Harding AE (1983) Classification of the hereditary ataxias and paraplegias. Lancet 1(8334):1151-1155

2. Duenas AM, Goold R, Giunti P (2006) Molecular pathogenesis of spinocerebellar ataxias. Brain 1029(Pt 6):1357-1370

3. Dudding TE, Friend K, Schofield PW, Lee S, Wilkinson IA, Richards RI (2004) Autosomal dominant congenital non-progressive ataxia overlaps with the SCA15 locus. Neurology 63 (12):2288-2292

4. Ishikawa K, Toru S, Tsunemi T, Li M, Kobayashi K, Yokota T et al (2005) An autosomal dominant cerebellar ataxia linked to chromosome $16 \mathrm{q} 22.1$ is associated with a single-nucleotide substitution in the $5^{\prime}$ untranslated region of the gene encoding a protein with spectrin repeat and Rho guanine-nucleotide exchangefactor domains. Am J Hum Genet 77(2):280-296

5. Houlden H, Johnson J, Gardner-Thorpe C, Lashley T, Hernandez D, Worth P et al (2007) Mutations in TTBK2, encoding a kinase implicated in tau phosphorylation, segregate with spinocerebellar ataxia type 11. Nat Genet 39(12):1434-1436

6. van de Leemput J, Chandran J, Knight MA, Holtzclaw LA, Scholz S, Cookson MR et al (2007) Deletion at ITPR1 underlies ataxia in mice and spinocerebellar ataxia 15 in humans. PLoS Genet 3(6):e108

7. Mariotti C, Brusco A, Di Bella D, Cagnoli C, Seri M, Gellera C et al (2008) Spinocerebellar ataxia type 28: a novel autosomal dominant cerebellar ataxia characterized by slow progression and ophthalmoparesis. Cerebellum 7(2):184-188

8. Knight MA, Hernandez D, Diede SJ, Dauwerse HG, Rafferty I, van de Leemput $J$ et al (2008) A duplication at chromosome 11q12.2-11q12.3 is associated with Spinocerebellar Ataxia Type 20 (SCA20). Hum Mol Genet doi:10.1093/hmg/ddn283

9. van de Warrenburg BP, Sinke RJ, Verschuuren-Bemelmans CC, Scheffer H, Brunt ER, Ippel PF et al (2002) Spinocerebellar ataxias in the Netherlands: prevalence and age at onset variance analysis. Neurology 58(5):702-708

10. Silveira I, Miranda C, Guimaraes L, Moreira MC, Alonso I, Mendonca $\mathrm{P}$ et al (2002) Trinucleotide repeats in 202 families with ataxia: a small expanded (CAG)n allele at the SCA17 locus. Arch Neurol 59(4):623-629

11. Schols L, Bauer P, Schmidt T, Schulte T, Riess O (2004) Autosomal dominant cerebellar ataxias: clinical features, genetics, and pathogenesis. Lancet Neurol 3(5):291-304

12. Bryer A, Krause A, Bill P, Davids V, Bryant D, Butler J et al (2003) The hereditary adult-onset ataxias in South Africa. J Neurol Sci 216(1):47-54

13. Saleem Q, Choudhry S, Mukerji M, Bashyam L, Padma MV, Chakravarthy A et al (2000) Molecular analysis of autosomal dominant hereditary ataxias in the Indian population: high frequency of SCA2 and evidence for a common founder mutation. Hum Genet 106(2):179-187

14. Brusco A, Gellera C, Cagnoli C, Saluto A, Castucci A, Michielotto C et al (2004) Molecular genetics of hereditary spinocerebellar ataxia: mutation analysis of spinocerebellar ataxia genes and $\mathrm{CAG} / \mathrm{CTG}$ repeat expansion detection in 225 Italian families. Arch Neurol 61(5):727-733

15. Verbeek DS, van de Warrenburg BP, Wesseling P, Pearson PL, Kremer HP, Sinke RJ (2004) Mapping of the SCA23 locus involved in autosomal dominant cerebellar ataxia to chromosome region 20p13-12.3. Brain 127(Pt 11):2551-2557

16. Lim J, Hao T, Shaw C, Patel AJ, Szabo G, Rual JF et al (2006) A protein-protein interaction network for human inherited ataxias and disorders of Purkinje cell degeneration. Cell 125(4):801-814

17. Franke L, Bakel H, Fokkens L, de Jong ED, Egmont-Petersen M, Wijmenga C (2006) Reconstruction of a functional human gene network, with an application for prioritizing positional candidate genes. Am J Hum Genet 78(6):1011-1025 